Monday, $2 d$ February 1846.

Sir T. M. BRISBANE, Bart., President, in the Chair.

The following Communications were read:-

\title{
1. On the Decomposition and Dispersion of Light within
} Solid Bodies. By Sir David Brewster, K.H.

After noticing various cases of solid and liquid bodies, which disperse light of a colour quite different from the transmitted tint, and Sir John Herschel's explanation of the phenomenon in the solution of acid sulphate of quinine, which, although itself colourless, disperses light of a bright blue, the author considers-

1. The internal dispersion of fluor-spar. By employing a more intense light than Sir J. Herschel did-namely, a condensed beam of the sun's light-he conceives that he has proved that the dispersion is not, as Sir J. Herschel supposes, superficial or epipolic, but, on the contrary, belongs, in some specimens at least, to every part of the crystal. The phenomenon is only seen in the green fluor of Alston, and in some pink and bluish-yellów varieties from Derbyshire. Some specimens are formed of strata alternately dispersing and non-dispersing. The author has not found the same appearances in any other mineral; but he has observed similar phenomena of dispersion in several kinds of glass, and even in some colourless glasses, which disperse a fine green tint.

2. On the internal dispersion of the acid solution of sulphate of quinine. Here, also, by using a more intense light than Sir J. Herschel, the author establishes that the peculiar dispersion occurs, not only in a stratum 1-50th of an inch thick at the surface of the liquid, but at all parts of it; and he concludes - First, That a beam of light, epipolised by the action of a solid or a liquid, is capable of further dispersion, provided the thickness of the medium has not been sufficient to disperse all the dispersible rays. Secondly, When such a medium is thus rendered incapable of dispersing more light, it is not, as Sir J. Herschel supposes, because the light has lost a property which it previously possessed, but because it has been deprived of all the dispersible rays it contained.

The rays thus dispersed are few in number, and, by their mixture, yield blue light, but they extend over a great range of refrangibility; while other rays, equally refrangible, are either less dispersible, or not dispersible. But this appears less surprising when we advert to the phenomena of absorption. 
The difference between absorption and dispersion is, that, in the former case, the absorbed light is extinguished; while, in the latter, the dispersed light is visible. Hence, if, in powerfully absorbing bodies, the absorbed light could be rendered visible, we should have the phenomena of epipolised light.

3. On the polarisation of dispersed light. Sir J. Herschel had failed to detect the polarisation of epipolised light; but the author, by using a condensed sunbeam, discovered that the dispersed beam was polarised, partly in the plane of reflexion, and partly in a peculiar manner; which the author calls quaquaversus polarisation, like that effected by a congeries of small doubly-refracting crystals, having their axes in all directions. He afterwards discovered instances in which each of these kinds of polarisation was found alone. These experiments have led to more extended investigations, to be subsequently communicated. The remainder of the section contains a minute description of the beautiful phenomena of the polarisation of dispersed light. The author thinks it probable that the study of these phenomena may throw much light on the internal structure of the substances exhibiting them.

4. On the causes of the decomposition and internal dispersion of light. The author ascribes the phenomena (which do not, in fluorspar, belong to the species, but only to certain varieties) to irregular crystallisation, and the successive deposition of strata of different refractive and dispersive power. He does not, at present, offer any theory of the cause in liquids.

2. A few Remarks suggested by Professor Forbes's Description of the effects of Glacial Action among the Cuchullin Hills, and Mr Maclaren's views of the facts observed by him at the Gareloch. By Sir G. S. Mackenzie, Bart.

The object of this paper was to shew, that if it be assumed that glaciers had actually existed among the Cuchullin Hills, or in other parts of this country, one of two conditions must necessarily have been present-either a climate much colder than the existing climate of Scotland, or a higher position of the land where the supposed marks of glaciers are seen. With respect to the first condition, the permanency of the relative positions of the heavenly bodies, and of the inclination of the earth's axis, and the adaptation to existing climates of animal and regetable life, were appealed to

VOL. II. 